Pacific

Journal of

Mathematics

NOTES ON THE EXTENSION OF THE MEAN CURVATURE FLOW

Yan Leng, Entao Zhao AND HaORan ZhaO

Volume 269 No. 2

June 2014 


\title{
NOTES ON THE EXTENSION OF THE MEAN CURVATURE FLOW
}

\author{
YAN LENG, ENTAO ZHAO AND HAORAN ZHAO
}

\begin{abstract}
In this paper, we present several new curvature conditions that assure the extension of the mean curvature flow on a finite time interval, which improve some known extension theorems.
\end{abstract}

\section{Introduction}

Let $F_{0}: M^{n} \rightarrow N^{n+d}$ be a smooth isometric immersion from an $n$-dimensional closed (compact and without boundary) Riemannian manifold $M$ to an $(n+d)$ dimensional Riemannian manifold $N$. Consider a one-parameter family of smooth isometric immersions $F: M \times[0, T) \rightarrow N$ satisfying

$$
\left\{\begin{aligned}
\frac{\partial}{\partial t} F(x, t) & =H(x, t), \\
F(x, 0) & =F_{0}(x),
\end{aligned}\right.
$$

where $H(x, t)$ is the mean curvature vector of $F_{t}(M)$ and $F_{t}(x)=F(x, t)$. Set $M_{t}=F_{t}(M)$. We call $F: M \times[0, T) \rightarrow N$ the mean curvature flow with initial value $F_{0}: M \rightarrow N$.

The mean curvature flow is a (degenerate) quasilinear parabolic evolution equation, and one can obtain the short-time existence either by the Nash-Moser implicit function theorem or by the DeTurck trick to modify the mean curvature flow equation to a strongly parabolic equation. Without any special assumption on $M_{0}$, the mean curvature flow (1-1) will in general develop singularities in finite time, characterized by blowing up of the second fundamental form $A$.

Theorem 1.1 [Huisken 1984; 1986; Wang 2001]. Suppose $T<\infty$ is the first singular time for a closed mean curvature flow in a Riemannian manifold with bounded geometry. Then we have

$$
\lim _{t \rightarrow T} \sup _{M_{t}}|A|=\infty .
$$

Research supported by the National Natural Science Foundation of China, grants 11371315 and 11201416.

MSC2010: 53C44.

Keywords: Mean curvature flow, extension, blow-up method, volume expansion. 
From Theorem 1.1, we see that if $\sup _{M_{t} \times[0, T)}|A|$ is bounded, then the mean curvature flow can be extended past the time $T$. Recently, Le and Šešum [2011] and Liu, Xu, Ye and Zhao [Liu et al. 2011; Xu et al. 2011a; 2011b] obtained some integral conditions to extend the mean curvature flow. Define a $(0,2)$-tensor $B$ on $M$ in a local orthonormal frame field by $B_{i j}=\left\langle H, h_{i j}\right\rangle$. Cooper obtained the following characterization of the singular time.

Theorem 1.2 [Cooper 2011]. Suppose $T<\infty$ is the first singular time for a closed mean curvature flow in a Riemannian manifold with bounded geometry. Then we have

$$
\lim _{t \rightarrow T} \sup _{M_{t}}|B|=\infty
$$

Similarly, we see from Theorem 1.2 that if $\sup _{M_{t} \times[0, T)}|B|$ is bounded, then the mean curvature flow can be extended past the time $T$.

In the present paper, we make an improvement of Theorems 1.1 and 1.2 by considering the integral of $|B|$ on the time interval. More precisely, we prove the following theorem.

Theorem 1.3. Let $F_{t}: M^{n} \rightarrow N^{n+d}$ be the mean curvature flow solution of closed submanifolds on a finite time interval $[0, T)$ and assume $N$ has bounded geometry. If the function $f(x):=\int_{0}^{T}|B|(x, t) d t$ is continuous on $M$, then the mean curvature flow can be extended past the time $T$.

By the dominated convergence theorem and Theorem 1.3, we obtain the following result, which recovers Theorems 1.1 and 1.2.

Theorem 1.4. Suppose $T<\infty$ is the first singular time for a closed mean curvature flow in a Riemannian manifold with bounded geometry. Then we have

$$
\int_{0}^{T} \sup _{M_{t}}|B|(t) d t=\infty
$$

Analogous extension theorems for the Ricci flow have been proved recently [Wang 2012; He 2014]. Some general regularity results have been obtained by Cheeger, Haslhofer and Naber [Cheeger et al. 2013] and Ecker [2013], among others. To prove our theorems we combine the ideas in [Cooper 2011] and [He 2014]. First, by a suitable blow-up argument we get a minimal submanifold in Euclidean space. Second, we prove that the volume of geodesic balls in this minimal submanifold is less than the volume of geodesic balls with same radius. By the expansion for the volume of geodesic balls, we see that this minimal submanifold is in fact totally geodesic at the base point. 


\section{Preliminaries}

Let $M^{n}$ be an $n$-dimensional submanifold isometrically immersed in an $(n+d)$ dimensional Riemannian manifold $N^{n+d}$. Let $A$ and $H$ be the second fundamental form and the mean curvature vector of $M$ in $N$, respectively. Define a $(0,2)$ tensor $B$ from $A$ and $H$ by $B=\langle A, H\rangle$. Choose a local orthonormal frame field $\left\{e_{A}\right\}_{A=1}^{n+d}$ in $N^{n+d}$ such that each $e_{i}, i=1, \ldots, n$, is tangent to $M$ and let $\left\{\omega_{A}\right\}_{A=1}^{n+d}$ be the dual frame field of $\left\{e_{A}\right\}_{A=1}^{n+d}$. Then $A, H$ and $B$ can be written as

$$
\begin{aligned}
A & =\sum_{i, j=1}^{n} \sum_{\alpha=n+1}^{n+d} h_{i j}^{\alpha} \omega_{i} \otimes \omega_{j} \otimes e_{\alpha}=\sum_{i, j} h_{i j} \omega_{i} \otimes \omega_{j}, h_{i j}=\sum_{\alpha=n+1}^{n+d} h_{i j}^{\alpha} e_{\alpha}, \\
H & =\sum_{\alpha} H^{\alpha} e_{\alpha}, H^{\alpha}=\sum_{i=1}^{n} h_{i i}^{\alpha}, \\
B & =\sum_{i, j=1}^{n} B_{i j} \omega_{i} \otimes \omega_{j}, B_{i j}=\sum_{\alpha=n+1}^{n+d} H^{\alpha} h_{i j}^{\alpha} .
\end{aligned}
$$

Let $F: M \times[0, T) \rightarrow N$ be a mean curvature flow solution with initial immersion $F_{0}: M \rightarrow N$. Denote by $g(t)$ and $d \mu(t)$ the induced metric and the volume form on $M$. Under the mean curvature flow, $g(t)$ and $d \mu(t)$ satisfy the following evolution equations.

$$
\begin{gathered}
\frac{\partial}{\partial t} g(t)=-2 B(t), \\
\frac{\partial}{\partial t} d \mu(t)=-|H|^{2} d \mu(t) .
\end{gathered}
$$

\section{Proof of Theorem 1.3}

Now we give the proof of Theorem 1.3.

Proof. We argue by contradiction. Suppose that $T$ is the maximal existence time. Then by Theorem 1.1 we see that $\lim _{t \rightarrow T} \sup _{M_{t}}|A|=\infty$. Choose a sequence of points $\left(O_{i}, t_{i}\right) \in M \times[0, T), i=1,2, \ldots$, such that $\lim _{i \rightarrow \infty} t_{i}=T$ and

$$
|A|^{2}\left(O_{i}, t_{i}\right)=\max _{(x, t) \in M \times\left[0, t_{i}\right]}|A|^{2}(x, t) \rightarrow \infty \quad \text { as } i \rightarrow \infty .
$$

Set $Q_{i}=|A|^{2}\left(O_{i}, t_{i}\right)$ and we suppose $Q_{i} \geq 1$ and $Q_{i} t_{i} \geq 1$. Denote by $h$ the Riemannian metric on $N$. We consider the rescaled flows for $t \in[0,1]$

$$
F_{i}(t)=F\left(\frac{t-1}{Q_{i}}+t_{i}\right):\left(M, g_{i}(t)\right) \longrightarrow\left(N, Q_{i} h\right),
$$

where $g_{i}(t)=F_{i}(t)^{*}\left(Q_{i} h\right)$ is the induced metric on $M$. Then for each $i, F_{i}$ is also 
a solution of the mean curvature flow on time interval $[0,1]$. Denote by $M_{i}$ the manifold $M$ with metric $g_{i}(t)$. It follows from [Chen and He 2010] that there is a subsequence of $\left\{\left(M_{i}, g_{i}(t), O_{i}\right): i=1,2, \ldots\right\}$ which converges to a Riemannian manifold $\left(M_{\infty}, g_{\infty}(t), O_{\infty}\right)$, and the corresponding subsequence of immersions $F_{i}(t)$ converges to an immersion $F_{\infty}(t): M_{\infty} \rightarrow \mathbb{R}^{n+d}, t \in[0,1]$. Note that $F_{\infty}$ is also a solution of the mean curvature flow on time interval $[0,1]$.

We first show that for any $t \in[0,1], M_{\infty}$ is a minimal submanifold in $\mathbb{R}^{n+d}$. Let $B_{\infty}(\cdot, t)$ be the $(0,2)$-tensor for $F_{\infty}(t)$. In fact, we prove the following:

Lemma 3.1.

$$
B_{\infty}(t)=0 \quad \text { for } t \in[0,1] .
$$

Proof. By the continuity assumption on

$$
f(x):=\int_{0}^{T}|B|(x, t) d t
$$

and the compactness of $M$, we can use elementary arguments to prove that

$$
\lim _{t \rightarrow T} \int_{t}^{T}|B|(x, t) d t=0 .
$$

First, we have

$$
\begin{aligned}
g_{i}(t) & =F_{i}(t)^{*}\left(Q_{i} h\right)=F\left(\frac{t-1}{Q_{i}}+t_{i}\right)^{*}\left(Q_{i} h\right) \\
& =Q_{i} F\left(\frac{t-1}{Q_{i}}+t_{i}\right)^{*}(h)=Q_{i} g\left(\frac{t-1}{Q_{i}}+t_{i}\right) .
\end{aligned}
$$

Denote by $A_{i}(\cdot, t), H_{i}(\cdot, t)$ and $B_{i}(\cdot, t)$ the second fundamental form, the mean curvature and the $(0,2)$-tensor of $F_{i}(t)$, respectively. It is easy to see from the definition of second fundamental form that

$$
A_{i}(\cdot, t)=A\left(\cdot, \frac{t-1}{Q_{i}}+t_{i}\right) .
$$

Since the mean curvature is the trace of the second fundamental form, we have

$$
H_{i}(\cdot, t)=Q_{i}^{-1} H\left(\cdot, \frac{t-1}{Q_{i}}+t_{i}\right) .
$$

So for the $(0,2)$-tensor we have

$$
\begin{aligned}
B_{i}(\cdot, t) & =\left\langle A_{i}(\cdot, t), H_{i}(\cdot, t)\right\rangle_{Q_{i} h} \\
& =\left\langle A\left(\cdot, \frac{t-1}{Q_{i}}+t_{i}\right), H\left(\cdot, \frac{t-1}{Q_{i}}+t_{i}\right)\right\rangle_{h}=B\left(\cdot, \frac{t-1}{Q_{i}}+t_{i}\right) .
\end{aligned}
$$


From this we see that

$$
\begin{aligned}
\left|B_{i}(\cdot, t)\right|_{g_{i}(t)}^{2} & =\left\langle B_{i}(\cdot, t), B_{i}(\cdot, t)\right\rangle_{g_{i}(t) \otimes g_{i}(t)} \\
& =Q_{i}^{-2}\left\langle B\left(\cdot, \frac{t-1}{Q_{i}}+t_{i}\right), B\left(\cdot, \frac{t-1}{Q_{i}}+t_{i}\right)\right\rangle_{g\left((t-1) / Q_{i}+t_{i}\right) \otimes g\left((t-1) / Q_{i}+t_{i}\right)} \\
& =Q_{i}^{-2}\left|B\left(\cdot, \frac{t-1}{Q_{i}}+t_{i}\right)\right|_{g\left((t-1) / Q_{i}+t_{i}\right)}^{2} .
\end{aligned}
$$

For any $y \in M_{\infty}$, there are $y_{i} \in M, i=1,2, \ldots$, such that $\lim _{i \rightarrow \infty} y_{i}=y$.

$$
\begin{aligned}
\int_{0}^{1}|B|_{g_{\infty}(t)}(y, t) d t & =\lim _{i \rightarrow \infty} \int_{0}^{1}\left|B_{i}\right|_{g_{i}(t)}\left(y_{i}, t\right) d t \\
& =\lim _{i \rightarrow \infty} Q_{i}^{-1} \int_{0}^{1}|B|_{g\left((t-1) / Q_{i}+t_{i}\right)}\left(y_{i}, \frac{t-1}{Q_{i}}+t_{i}\right) d t \\
& =\lim _{i \rightarrow \infty} \int_{t_{i}-Q_{i}^{-1}}^{t_{i}}|B|_{g(s)}\left(y_{i}, s\right) d s \\
& =0 .
\end{aligned}
$$

Hence we have $B_{\infty}(t)=0$ for each $t \in[0,1]$.

Lemma 3.2. The induced metrics $g(t)$ on $M$ are uniformly equivalent and converge pointwise as $t \rightarrow T$ to a continuous positive-definite metric $g(T)$.

Proof. Under the assumption that $f(x)=\int_{0}^{T}|B|(x, t) d t$ is continuous, we see that $f(x)$ is bounded and for any $0 \leq \tau \leq \theta<T$

$$
\lim _{\tau \rightarrow \theta} \int_{\tau}^{\theta}|B|(x, t) d t=0
$$

uniformly. Since $g(t)$ satisfies (2-1), we can carry out the same argument as in [Hamilton 1982] to prove the lemma.

Let $\boldsymbol{B}_{g_{\infty}(1)}\left(O_{\infty}, r\right)$ be the geodesic ball of radius $r$ centered at $O_{\infty} \in M_{\infty}$ with respect to the metric $g_{\infty}(1)$, and $\operatorname{Vol}_{g_{\infty}(1)}\left(\boldsymbol{B}_{g_{\infty}(1)}\left(O_{\infty}, r\right)\right)$ be the volume of $\boldsymbol{B}_{g_{\infty}(1)}\left(O_{\infty}, r\right)$. Denote by $\omega_{n}$ the volume of the unit ball in $\mathbb{R}^{n}$.

$$
\text { Lemma 3.3. } \quad \operatorname{Vol}_{g_{\infty}(1)}\left(\boldsymbol{B}_{g_{\infty}(1)}\left(O_{\infty}, r\right)\right) \leq \omega_{n} r^{n} \text {. }
$$

Proof. Let $\boldsymbol{B}_{g_{i}(1)}\left(O_{i}, r\right)$ be the geodesic ball with radius $r$ centered at $O_{i} \in M_{i}$ with respect to $g_{i}(t)$ and $\operatorname{Vol}_{g_{i}(1)}\left(\boldsymbol{B}_{g_{i}(1)}\left(O_{i}, r\right)\right)$ the volume of $\boldsymbol{B}_{g_{i}(1)}\left(O_{i}, r\right)$. It is easy to see that

$$
\boldsymbol{B}_{g_{i}(1)}\left(O_{i}, r\right)=\boldsymbol{B}_{g\left(t_{i}\right)}\left(O_{i}, Q_{i}^{-1 / 2} r\right)
$$


Hence

$$
\begin{aligned}
\frac{\operatorname{Vol}_{g_{\infty}(1)}\left(\boldsymbol{B}_{g_{\infty}(1)}\left(O_{\infty}, r\right)\right)}{r^{n}} & =\lim _{i \rightarrow \infty} \frac{\operatorname{Vol}_{g_{i}(1)}\left(\boldsymbol{B}_{g_{i}(1)}\left(O_{i}, r\right)\right)}{r^{n}} \\
& =\lim _{i \rightarrow \infty} \frac{\operatorname{Vol}_{g_{i}(1)}\left(\boldsymbol{B}_{g\left(t_{i}\right)}\left(O_{i}, Q_{i}^{-1 / 2} r\right)\right)}{r^{n}} \\
& =\lim _{i \rightarrow \infty} \frac{Q_{i}^{n / 2} \operatorname{Vol}_{g\left(t_{i}\right)}\left(\boldsymbol{B}_{g\left(t_{i}\right)}\left(O_{i}, Q_{i}^{-1 / 2} r\right)\right)}{r^{n}} \\
& =\lim _{i \rightarrow \infty} \frac{\operatorname{Vol}_{g\left(t_{i}\right)}\left(\boldsymbol{B}_{g\left(t_{i}\right)}\left(O_{i}, Q_{i}^{-1 / 2} r\right)\right)}{\left(Q_{i}^{-1 / 2} r\right)^{n}} .
\end{aligned}
$$

From Lemma 3.2, we see that for any $\varepsilon>0$, there is a positive constant $\delta$ such that if $t \geq t_{0}>T-\delta$, then $(1-\varepsilon) g\left(t_{0}\right) \leq g(t) \leq(1+\varepsilon) g\left(t_{0}\right)$. We may pick $t_{i}$ s such that $t_{i} \geq t_{0} \geq T-\delta$. From a lemma in [Cooper 2011; Glickenstein 2003] we see that

$$
\begin{aligned}
& \lim _{i \rightarrow \infty} \frac{\operatorname{Vol}_{g\left(t_{i}\right)}\left(\boldsymbol{B}_{g\left(t_{i}\right)}\left(O_{i}, Q_{i}^{-1 / 2} r\right)\right)}{\left(Q_{i}^{-1 / 2} r\right)^{n}} \\
& \leq \lim _{i \rightarrow \infty} \frac{\operatorname{Vol}_{g\left(t_{i}\right)}\left(\boldsymbol{B}_{g\left(t_{0}\right)}\left(O_{i},\left((1-\varepsilon) Q_{i}\right)^{-1 / 2} r\right)\right)}{\left(Q_{i}^{-1 / 2} r\right)^{n}} \\
& \leq \lim _{i \rightarrow \infty} \frac{\operatorname{Vol}_{g\left(t_{0}\right)}\left(\boldsymbol{B}_{g\left(t_{0}\right)}\left(O_{i},\left((1-\varepsilon) Q_{i}\right)^{-1 / 2} r\right)\right)}{\left(Q_{i}^{-1 / 2} r\right)^{n}} \\
& \quad=(1-\varepsilon)^{-n / 2} \lim _{i \rightarrow \infty} \frac{\operatorname{Vol}_{g\left(t_{0}\right)}\left(\boldsymbol{B}_{g\left(t_{0}\right)}\left(O_{i},\left((1-\varepsilon) Q_{i}\right)^{-1 / 2} r\right)\right)}{\left(\left((1-\varepsilon) Q_{i}\right)^{-1 / 2} r\right)^{n}} .
\end{aligned}
$$

Since $Q_{i} \rightarrow \infty$ as $i \rightarrow \infty$, we have

$$
\lim _{i \rightarrow \infty} \frac{\operatorname{Vol}_{g\left(t_{0}\right)}\left(\boldsymbol{B}_{g\left(t_{0}\right)}\left(O_{i},\left((1-\varepsilon) Q_{i}\right)^{-1 / 2} r\right)\right)}{\left(\left((1-\varepsilon) Q_{i}\right)^{-1 / 2} r\right)^{n}}=\omega_{n} .
$$

Since $\varepsilon$ is arbitrary, we see that

$$
\frac{\operatorname{Vol}_{g_{\infty}(1)}\left(\boldsymbol{B}_{g_{\infty}(1)}\left(O_{\infty}, r\right)\right)}{r^{n}} \leq \omega_{n} .
$$

We continue the proof of Theorem 1.3. From the expansion formula for the volume of small balls (see Theorem 3.98 of [Gallot et al. 1987]) we have

$$
\frac{\operatorname{Vol}_{g_{\infty}(1)}\left(\boldsymbol{B}_{g_{\infty}(1)}\left(O_{\infty}, r\right)\right)}{\omega_{n} r^{n}}=1-\frac{R\left(O_{\infty}\right)}{6(n+2)} r^{2}+o\left(r^{2}\right) .
$$

Here $R\left(O_{\infty}\right)$ is the scalar curvature at $O_{\infty} \in\left(M_{\infty}, g_{\infty}(1)\right)$. From Lemma 3.3 we see that

$$
R\left(O_{\infty}\right) \geq 0
$$


This combined with Lemma 3.1 implies that

$$
|A|_{\infty}\left(O_{\infty}, 1\right)=0 .
$$

However, it is seen from the point selecting process that

$$
|A|_{\infty}\left(O_{\infty}, 1\right)=1
$$

This is a contradiction, which completes the proof of Theorem 1.3.

Theorem 3.4. Let $F_{t}: M^{n} \rightarrow N^{n+d}$ be the mean curvature flow solution of closed submanifolds on a finite time interval $[0, T)$ and assume $N$ has bounded geometry. Suppose $T<\infty$ is the first singular time. If the function $\int_{0}^{T}|A|(x, t) d t<+\infty$ is continuous on $M$, then we have

$$
\lim _{t \rightarrow T} \sup _{M_{t}}|H|=\infty .
$$

Proof. We suppose $|H| \leq C$ uniformly for all the existence time. Then

$$
\left|\frac{\partial g}{\partial t}\right|=2|B| \leq 2|H||A| \leq 2 C|A| .
$$

By the dominated convergence theorem, we know that $\int_{0}^{T}|A|(x, t) d t$ is continuous in $x$. Then by a similar argument as in the proof of Theorem 1.3, we get the conclusion.

Remark 3.5. Theorem 3.4 recovers [Cooper 2011, Theorem 5.1].

\section{Acknowledgement}

The authors thank Professor Kefeng Liu and Professor Hongwei Xu for their help and discussions.

\section{References}

[Cheeger et al. 2013] J. Cheeger, R. Haslhofer, and A. Naber, "Quantitative stratification and the regularity of mean curvature flow", Geom. Funct. Anal. 23:3 (2013), 828-847. MR 3061773 Zbl 1277.53064

[Chen and He 2010] J. Chen and W. He, "A note on singular time of mean curvature flow", Math. Z. 266:4 (2010), 921-931. MR 2011j:53119 Zbl 1201.53075

[Cooper 2011] A. A. Cooper, "A characterization of the singular time of the mean curvature flow", Proc. Amer. Math. Soc. 139:8 (2011), 2933-2942. MR 2012d:53211 Zbl 1220.53080

[Ecker 2013] K. Ecker, "Partial regularity at the first singular time for hypersurfaces evolving by mean curvature", Math. Ann. 356:1 (2013), 217-240. MR 3038128 Zbl 1270.53084

[Gallot et al. 1987] S. Gallot, D. Hulin, and J. Lafontaine, Riemannian geometry, Springer, Berlin, 1987. MR 88k:53001 Zbl 0636.53001

[Glickenstein 2003] D. Glickenstein, "Precompactness of solutions to the Ricci flow in the absence of injectivity radius estimates", Geom. Topol. 7 (2003), 487-510. MR 2004k:53099 Zbl 1044.53048 
[Hamilton 1982] R. S. Hamilton, "Three-manifolds with positive Ricci curvature", J. Differential Geom. 17:2 (1982), 255-306. MR 84a:53050 Zbl 0504.53034

[He 2014] F. He, "Remarks on the extension of the Ricci flow", J. Geom. Anal. 24:1 (2014), 81-91. MR 3145915

[Huisken 1984] G. Huisken, "Flow by mean curvature of convex surfaces into spheres", J. Differential Geom. 20:1 (1984), 237-266. MR 86j:53097 Zbl 0556.53001

[Huisken 1986] G. Huisken, "Contracting convex hypersurfaces in Riemannian manifolds by their mean curvature”, Invent. Math. 84:3 (1986), 463-480. MR 87f:53066 Zbl 0589.53058

[Le and Šešum 2011] N. Q. Le and N. Sesum, "On the extension of the mean curvature flow", Math. Z. 267:3-4 (2011), 583-604. MR 2012h:53153 Zbl 1216.53060

[Liu et al. 2011] K. F. Liu, H.-W. Xu, F. Ye, and E.-T. Zhao, "The extension and convergence of mean curvature flow in higher codimension”, preprint, 2011. arXiv 1104.0971v1

[Wang 2001] M.-T. Wang, "Mean curvature flow of surfaces in Einstein four-manifolds", J. Differential Geom. 57:2 (2001), 301-338. MR 2003j:53108 Zbl 1035.53094

[Wang 2012] B. Wang, "On the conditions to extend Ricci flow(II)", Int. Math. Res. Not. 2012:14 (2012), 3192-3223. MR 2946223 Zbl 1251.53040

[Xu et al. 2011a] H.-W. Xu, F. Ye, and E.-T. Zhao, "Extend mean curvature flow with finite integral curvature", Asian J. Math. 15:4 (2011), 549-556. MR 2012h:53158 Zbl 1242.53085

[Xu et al. 2011b] H.-W. Xu, F. Ye, and E.-T. Zhao, "The extension for mean curvature flow with finite integral curvature in Riemannian manifolds", Sci. China Math. 54:10 (2011), 2195-2204. MR 2012k:53133 Zbl 1232.53056

Received March 25, 2013. Revised September 11, 2013.

YAN LENG

Center of Mathematical Sciences

ZHEJIANG UNIVERSITY

HANGZHOU, 310027

CHINA

lengyan@cms.zju.edu.cn

\author{
ENTAO ZHAO \\ Center of Mathematical SCiEnces \\ ZHEJIANG UNIVERSITY \\ HANGZHOU, 310027 \\ CHINA \\ zhaoet@cms.zju.edu.cn \\ HAORAN ZHAO \\ Center of Mathematical Sciences \\ ZHEJIANG UNIVERSITY \\ HANGZHOU, 310027 \\ CHINA \\ zhaohaoran2010@gmail.com
}




\title{
PACIFIC JOURNAL OF MATHEMATICS
}

\author{
msp.org/pjm
}

Founded in 1951 by E. F. Beckenbach (1906-1982) and F. Wolf (1904-1989)

\section{EDITORS}

Don Blasius (Managing Editor)

Department of Mathematics

University of California

Los Angeles, CA 90095-1555

blasius@math.ucla.edu

\author{
Paul Balmer \\ Department of Mathematics \\ University of California \\ Los Angeles, CA 90095-1555 \\ balmer@math.ucla.edu \\ Robert Finn \\ Department of Mathematics \\ Stanford University \\ Stanford, CA 94305-2125 \\ finn@math.stanford.edu \\ Sorin Popa \\ Department of Mathematics \\ University of California \\ Los Angeles, CA 90095-1555 \\ popa@math.ucla.edu
}

\author{
Vyjayanthi Chari \\ Department of Mathematics \\ University of California \\ Riverside, CA 92521-0135 \\ chari@math.ucr.edu \\ Kefeng Liu \\ Department of Mathematics \\ University of California \\ Los Angeles, CA 90095-1555 \\ liu@math.ucla.edu \\ Jie Qing \\ Department of Mathematics \\ University of California \\ Santa Cruz, CA 95064 \\ qing@ cats.ucsc.edu
}

\section{PRODUCTION}

Silvio Levy, Scientific Editor, production@msp.org

\section{SUPPORTING INSTITUTIONS}

ACADEMIA SINICA, TAIPEI

CALIFORNIA INST. OF TECHNOLOGY

INST. DE MATEMÁTICA PURA E APLICADA

KEIO UNIVERSITY

MATH. SCIENCES RESEARCH INSTITUTE

NEW MEXICO STATE UNIV.

OREGON STATE UNIV.

\author{
STANFORD UNIVERSITY \\ UNIV. OF BRITISH COLUMBIA \\ UNIV. OF CALIFORNIA, BERKELEY \\ UNIV. OF CALIFORNIA, DAVIS \\ UNIV. OF CALIFORNIA, LOS ANGELES \\ UNIV. OF CALIFORNIA, RIVERSIDE \\ UNIV. OF CALIFORNIA, SAN DIEGO \\ UNIV. OF CALIF., SANTA BARBARA
}

\author{
Daryl Cooper \\ Department of Mathematics \\ University of California \\ Santa Barbara, CA 93106-3080 \\ cooper@math.ucsb.edu \\ Jiang-Hua Lu \\ Department of Mathematics \\ The University of Hong Kong \\ Pokfulam Rd., Hong Kong \\ jhlu@maths.hku.hk \\ Paul Yang \\ Department of Mathematics \\ Princeton University \\ Princeton NJ 08544-1000 \\ yang@math.princeton.edu
}

These supporting institutions contribute to the cost of publication of this Journal, but they are not owners or publishers and have no responsibility for its contents or policies.

See inside back cover or msp.org/pjm for submission instructions.

The subscription price for 2014 is US $\$ 410 /$ year for the electronic version, and \$535/year for print and electronic.

Subscriptions, requests for back issues and changes of subscribers address should be sent to Pacific Journal of Mathematics, P.O. Box 4163, Berkeley, CA 94704-0163, U.S.A. The Pacific Journal of Mathematics is indexed by Mathematical Reviews, Zentralblatt MATH, PASCAL CNRS Index, Referativnyi Zhurnal, Current Mathematical Publications and Web of Knowledge (Science Citation Index).

The Pacific Journal of Mathematics (ISSN 0030-8730) at the University of California, c/o Department of Mathematics, 798 Evans Hall \#3840, Berkeley, CA 94720-3840, is published twelve times a year. Periodical rate postage paid at Berkeley, CA 94704, and additional mailing offices. POSTMASTER: send address changes to Pacific Journal of Mathematics, P.O. Box 4163, Berkeley, CA 94704-0163.

PJM peer review and production are managed by EditFLOW ${ }^{\circledR}$ from Mathematical Sciences Publishers.

\section{PUBLISHED BY}

\section{mathematical sciences publishers \\ nonprofit scientific publishing}

http://msp.org/

(C) 2014 Mathematical Sciences Publishers 


\section{PACIFIC JOURNAL OF MATHEMATICS}

Volume $269 \quad$ No. $2 \quad$ June 2014

Totaro's question for simply connected groups of low rank

257

JODI BLACK and RAMAN PARIMALA

Uniform hyperbolicity of the curve graphs

269

BRIAN H. BOWDITCH

Constant Gaussian curvature surfaces in the 3-sphere via loop groups

281

DAVID BRANDER, JUN-ICHI INOGUCHI and SHIMPEI KOBAYASHI

On embeddings into compactly generated groups

305

PIERRE-EMmanuel CAPRACE and Yves CORNULIER

Variational representations for $N$-cyclically monotone vector fields

ALFRED GALICHON and NASSIF GHOUSSOUB

Restricted successive minima

341

MaRTIN HenK and CARSTEN THIEL

Radial solutions of non-Archimedean pseudodifferential equations

355

ANATOLY N. KochubeI

A Jantzen sum formula for restricted Verma modules over affine Kac-Moody

algebras at the critical level

JOHANNES KÜBEL

Notes on the extension of the mean curvature flow

YAN LENG, ENTAO ZHAO and HaORAN ZHAO

Hypersurfaces with prescribed angle function

Henrique F. DE Lima, ERAldo A. Lima JR. and Ulisses L. Parente

Existence of nonparametric solutions for a capillary problem in warped products

JoRGE H. LiRA and GABRIELA A. WANDERLEY

A counterexample to the simple loop conjecture for $\operatorname{PSL}(2, \mathbb{R})$

425

KATHRYN MANN

Twisted Alexander polynomials of 2-bridge knots for parabolic representations

TAKAYUKI MORIFUJI and ANH T. TRAN

Schwarzian differential equations associated to Shimura curves of genus zero

FANG-TING TU

Polynomial invariants of Weyl groups for Kac-Moody groups 